\title{
Philip Ording: 99 Variations on a Proof
}

\section{Princeton University Press 2019, XI + 160 Seiten, ISBN: 978-0-691-15883-9, €20,99}

\section{Joachim Hilgert}

Angenommen: 13. Januar 2021 / Online publiziert: 26. Januar 2021

(C) Der/die Autor(en) 2021

Der Held dieses feuilletonistischen Mathematikbuches ist die kubische Gleichung

$$
x^{3}-6 x^{2}+11 x-6=2 x-2 .
$$

Die 99 Beweisvariationen liefern alle Aussagen über diese Gleichung und ihre Lösungsmenge. Versteht man die Gleichung als Polynomgleichung für ganze, reelle oder komplexe Zahlen und errät, dass 1 eine Lösung ist, so liefert die Polynomdivision durch $x-1$, dass $x=4$ die einzige weitere Lösung ist. Die erste Beweisvariation besteht denn auch in der Umformung zu $x^{3}-6 x^{2}+9 x-4=0$ und der Feststellung $x^{3}-6 x^{2}+9 x-4=(x-1)^{2}(x-4)$.

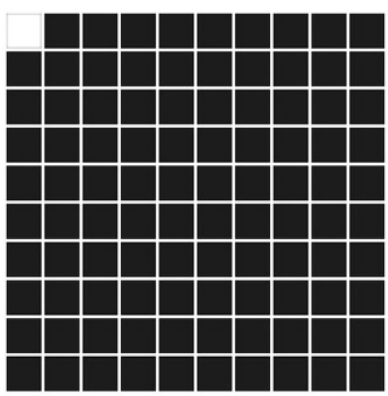

99 Variations on a Proof Philip Ording

Es kann dem Autor also nicht darum gehen, durch Variationen des Beweises mehr Licht in die Struktur der Gleichung und ihrer Lösungsmenge zu bringen. Er nimmt die einzelnen Variationen vielmehr zum Anlass für diverse Anmerkungen zu Natur und Kultur der Mathematik.

$\mathrm{Zu}$ Beginn geht es mehr um die Präzision in der Beschreibung von zu beweisender Aussage und einzelnen Beweisschritten. Die unterschiedlichen Beweise betonen unterschiedliche Aspekte der Argumentation, verwenden unterschiedliche Darstellungsformen, gehen unterschiedlich detailliert auf verwendete Rechengesetze ein. Im Laufe des

\footnotetext{
J. Hilgert $(\bowtie)$

Universität Paderborn, Paderborn, Deutschland

E-Mail: hilgert@upb.de
} 
Buches thematisiert der Autor immer wieder die Frage des Stils beim Schreiben mathematischer Texte - laut Vorwort die zentrale Motivation für das ganze Buch ${ }^{1}$.

Etliche Variationen illustrieren unterschiedliche Beweistechniken, wie zum Beispiel Variation 13 (Reductio ad absurdum), Variation 14 (Contrapositive), Variation 23 (Symmetry) und Variation 58 (Inventor's Paradox). Letzteres besagt, dass es manchmal leichter ist, eine schärfere Aussage zu zeigen als das ursprüngliche Problem zu lösen.

Viele der Variationen sind eigentlich keine Variationen des Beweises, sondern Variationen der zu beweisenden Aussage. Hier ein Beispiel - Variation 5 (Puzzle):

Suppose that among four consecutive numbers, the product of the first three equals the third. What's the fourth number?

Variation 7 (Found) ist der Scan einer Seite aus CARDANOs Ars Magna, in der 1545 zum ersten Mal die Lösungsformel für allgemeine kubische Gleichungen publiziert wurde.

Andere Variationen folgen dem Schema ,im Stile von ...“, wie zum Beispiel Variation 11 (Exam), Variation 34 (Medieval), Variation 43 (Screenplay) und Variation 81 (Doggerel).

Wieder eine andere Klasse von Variationen ist geometrischer Natur. Sei es, dass die algebraischen Terme als Volumina interpretiert werden wie in Variation 10 (Wordless), sei es, dass die Lösungen als Längen mit Zirkel und Lineal konstruiert werden wie in Variation 12 (Ruler and Compass), sei es, dass die Lösungen als Steigungen von Falzgeraden in Papierfaltungen produziert werden wie in Variation 39 (Origami).

Es gibt auch zwei Variationen, die mit Wahrscheinlichkeiten arbeiten: Variation 69 (Statistical) und Variation 78 (Probabilistic). Bezeichnenderweise liefern diese Beweisvarianten nur Abschätzungen für die Lösungen.

Wollte man die Variationen als Mathematiker nach ihrem mathematischen Gehalt und der Angemessenheit der Darstellung beurteilen, fiele das Urteil bisweilen harsch aus. Da werden für das eigentliche Problem unnötige Annahmen aus dem Hut gezaubert, um die Methode der Wahl überhaupt anwenden zu können und damit grobe Ergebnisse erzielt - besonders deutlich in Variation 69 (Statistics). Oder es wird mit Kanonen auf Spatzen geschossen, wie in den Variationen 61 (Modern) und 64 (Research Seminar). Aber eine solche Kritik wäre verfehlt. Der Autor gibt zu jeder Variation eine kurze Erläuterung, in der ein Phänomen beschrieben wird, das man anhand der gegebenen Variation illustrieren kann. Dieses Phänomen ist oft die eigentliche Motivation der entsprechenden Variation. Das Phänomen kann mathematischer Natur sein (zum Beispiel eine Beweistechnik). Es kann aber auch eine in der Mathematik übliche Lehrform (Variation 64) sein oder eine in der Community übliche Unart wie in Variation 94 (Authority), in der der proof by intimidation

\footnotetext{
1 Ording nennt das Buch Exercises in Style des französischen Schriftstellers und Mathematikliebhabers Raymond QUENEAU (1903-1976) als Vorbild. Da es erstaunlicherweise in der Literaturliste von 99 Variations on a Proof fehlt, hier die Erscheinungsdaten:

Französisches Original: Exercises de Style, Gallimard 1947.

Englische Übersetzung: Exercises in Style, Alma Classics, 2013.

Deutsche Übersetzung: Stilübungen. Suhrkamp, 1961.
} 
beschrieben wird. Es gibt auch Variationen, die ganz offensichtlich in erster Linie dazu da sind, einen geschichtlichen Exkurs unterzubringen, wie Variation 71 (Blog) mit seinen Erläuterungen zur persisch-arabischen Mathematik. Nicht zuletzt findet man Variationen, die den Humor des Autors widerspiegeln wie Variation 66 (Hand Waving). Hier sehen wir den Autor, wie er mit dem Finger eine kubische Kurve in die Luft malt. Die Erläuterung zu dieser Variation geht vom proof by hand waving aus, der einem in manchen Vorträgen begegnet, und spannt den Bogen zur Kognitionswissenschaft.

Insgesamt ist es Philip Ording gelungen, mit der kubischen Gleichung $x^{3}-6 x^{2}+$ $11 x-6=2 x-2$ einen roten Faden in sein mathematisches Kaleidoskop einzuweben, der die 100 Kalendergeschichten - Variation 0 (Omitted) wird auch erläutert - zu einer Einheit macht. Das Buch ist unterhaltsam, informativ und spricht Profis wie Laien an. Ich kann es nur empfehlen.

Funding Open Access funding enabled and organized by Projekt DEAL.

Open Access Dieser Artikel wird unter der Creative Commons Namensnennung 4.0 International Lizenz veröffentlicht, welche die Nutzung, Vervielfältigung, Bearbeitung, Verbreitung und Wiedergabe in jeglichem Medium und Format erlaubt, sofern Sie den/die ursprünglichen Autor(en) und die Quelle ordnungsgemäß nennen, einen Link zur Creative Commons Lizenz beifügen und angeben, ob Änderungen vorgenommen wurden.

Die in diesem Artikel enthaltenen Bilder und sonstiges Drittmaterial unterliegen ebenfalls der genannten Creative Commons Lizenz, sofern sich aus der Abbildungslegende nichts anderes ergibt. Sofern das betreffende Material nicht unter der genannten Creative Commons Lizenz steht und die betreffende Handlung nicht nach gesetzlichen Vorschriften erlaubt ist, ist für die oben aufgeführten Weiterverwendungen des Materials die Einwilligung des jeweiligen Rechteinhabers einzuholen.

Weitere Details zur Lizenz entnehmen Sie bitte der Lizenzinformation auf http://creativecommons.org/ licenses/by/4.0/deed.de. 